\title{
Überzeugungen und Belastungen durch Stimmenhören: die deutsche Version des Beliefs About Voices Questionnaire - Revised (BAVQ-R)
}

\author{
Andrea Gmeiner (D) - Jessica Aslan · Amy Gaglia · Tamara Rumpold · Beate Schrank · Stefanie Süßenbacher · \\ Michaela Amering
}

Eingegangen: 18. August 2018 / Angenommen: 18. September 2018 / Online publiziert: 28. September 2018 (C) Der/die Autor(en) 2018

\section{Zusammenfassung}

Hintergrund Überzeugungen zum Stimmenhören sind ein wesentlicher Faktor für Belastung, Coping und therapeutische Interventionen bei verbalen akustischen Halluzinationen. Ziel dieser Studie war es, den international etablierten Beliefs About Voices Questionnaire-Revised (BAVQ-R) in einer deutschen Übersetzung bei Personen mit Psychosen aus dem schizophrenen Formenkreis einzusetzen und zu validieren.

Methoden 93 Personen mit Erkrankungen aus dem schizophrenen Formenkreis und aktuellen verbalen akustischen Halluzinationen füllten den BAVQ-R für ihre dominante Stimme selbstständig aus. Weiters wurden soziodemographische und klinische $\mathrm{Da}$ ten sowie Daten zum Schweregrad der Erkrankung (Clinical Global Impression-Schizophrenie) und symptomspezifische Dimensionen des Stimmenhörens (Psychotic Symptoms Rating Scale) erhoben. Konstruktvalidität, Reliabilität und Test-Retest-Reliabilität wurden berechnet.

Ergebnisse Alle BAVQ-R Subskalen (Böswilligkeit, Wohlwollen, Widerstand, Bindung) außer der Sub-

Dr. med. univ. A. Gmeiner $(\bowtie) \cdot$ J. Aslan · T. Rumpold

S. Süßenbacher $\cdot$ M. Amering

Klinische Abteilung für Sozialpsychiatrie, Universitätsklinik

für Psychiatrie und Psychotherapie, Medizinische

Universität Wien, Währinger Gürtel 18-20, 1090 Wien,

Österreich

andrea.gmeiner@meduniwien.ac.at

A. Gaglia

Abteilung für Psychologie, Bangor University,

Bangor, Wales, Großbritannien

B. Schrank

Universitätsklinik Tulln, Abteilung für

Erwachsenenpsychiatrie, Karl Landsteiner Universität für

Gesundheitswissenschaften, Tulln an der Donau, Österreich skala Omnipotenz zeigten zufriedenstellende interne Konsistenz. Gute Test-Retest-Reliabilität ergab sich in den Überzeugungssubskalen (Böswilligkeit, Wohlwollen, Omnipotenz), aber nicht in Emotionen und Verhalten im Hinblick auf Widerstand und Bindung. Die signifikanten Korrelationen zwischen den Subskalen zeigten Ergebnisse, vergleichbar mit den jenen aus der Originalstudie des BAVQ-R. Es ergaben sich keine Korrelationen mit dem Schweregrad der Erkrankung, sondern mit Ausmaß und Intensität der Belastung durch Stimmen sowie deren negativen Inhalten.

Fazit für die Praxis Die einzuschätzenden Dimensionen bilden relevante Aspekte des subjektiven Erlebens des Stimmenhörens ab. Sowohl aus therapeutischer Sicht als auch als Forschungsbedarf stellt sich weiterhin aktuell die Frage nach der Konzeptualisierung vom Zusammenhang zwischen Kognition, Emotion und Verhalten.

Schlüsselwörter Psychometrie - Verbale akustische Halluzinationen · BAVQ-R · Validierung · Schizophrenie

\section{Beliefs and distress related to hearing voices: the German version of the Beliefs About Voices Questionnaire-Revised (BAVQ-R)}

\section{Summary}

Background Beliefs about voices are a determining factor with regards to distress, coping and therapeutic interventions in verbal auditory hallucinations. Aim of the present study was to use and validate the internationally well established Beliefs About Voices Questionnaire-Revised (BAVQ-R) in a German translation with people suffering from schizophrenic psychoses. Methods 93 participants with a diagnosis of schizophrenia and current verbal auditory hallucinations completed the BAVQ-R autonomously for their dominant 
voice. Sociodemographic and clinical data as well as data concerning the severity of illness (CGI-Sch) and symptom specific dimensions of voice-hearing (PSYRATS-AH) were collected. Construct validity, reliability and test-retest-reliability were calculated. Results Each of the subscales (malevolence, benevolence, resistance, engagement) with the exception of the subscale omnipotence showed satisfying internal consistency. Good test-retest-reliability was shown for the belief-subscales but not for emotion and behaviour in resistance and engagement. Significant correlations between the subscales showed the expected results, comparable to the results of the original BAVQ-R study. No correlations with severity of the illness, but with amount and intensity of distress through voices and their negative contents correlations were found.

Conclusions for practice The BAVQ-R is a feasible instrument to collect data about beliefs about voices in a valid and reliable way in people with a diagnosis of schizophrenia. From a therapeutic as well as from a scientific point of view, the question about the conceptualization of the relationship between cognition, emotion and behaviour stays relevant.

Keywords Psychometrics - Verbal auditory hallucinations $\cdot$ BAVQ-R $\cdot$ Validation $\cdot$ Schizophrenia

\section{Einleitung}

Der Beliefs About Voices Questionnaire - Revised [1] (BAVQ-R), die überarbeitete Version des BAVQ [2], ist ein Fragebogen, der Überzeugungen und emotionale und Verhaltensreaktionen von Personen bezüglich ihrer verbalen akustischen Halluzinationen erfasst. Im Rahmen der Entwicklung des bis heute wegweisenden kognitiven Modells des persistierenden Stimmenhörens [3] wurde der Fragebogen 1995 von Chadwick und Birchwood in einer Interviewstudie [2] mit 60 stimmenhörenden Personen mit Diagnosen einer Schizophrenie oder schizo-affektiven Störung kreiert. Die revidierte Fassung aus dem Jahr 2000 [1] verfeinerte die Beurteilungsmöglichkeiten und trug den Ergebnissen zur Bedeutung von Überzeugungen zur Omnipotenz der Stimmen mit zusätzlichen Items Rechnung. Es resultierten zufriedenstellende psychometrische Daten in einer Stichprobe von 71 stimmenhörenden Personen mit F2 und F3 Diagnosen.

Der BAVQ-R [1] kommt im englischsprachigen Raum und international sowohl in der klinischen Arbeit als auch in der Forschung zum Einsatz, da sich wiederholt gezeigt hat, dass Überzeugungen zum Stimmenhören die damit verbundenen Belastungen und die affektiven und Verhaltensreaktionen auf dieses Symptom erheblich prägen [3]. Der BAVQ-R mit seinen fünf Subskalen Böswilligkeit, Wohlwollen, Omnipotenz, Widerstand und Bindung (jeweils Emotion und Verhalten) dient der Evaluation von Interventio- nen [4, 5], der Beobachtung des Verlaufs [6] sowie der Forschung zur kognitiven Verhaltenstherapie, Compliance mit imperativen Stimmen und Unterschieden zwischen befehlenden und nicht-befehlenden Stimmen [7-9]. Er kann auch unabhängig von einer Schizophrenie-Diagnose, also z. B. bei PatientInnen mit Persönlichkeitsstörung vom Borderlinetyp und gesunden StimmenhörerInnen eingesetzt werden [10]. Hepworth et al. [11] untersuchten Personen mit schizophrenen Erkrankungen sowie Persönlichkeitsstörungen vom Borderlinetyp und fanden heraus, dass es keine Hinweise auf Unterschiedlichkeit in der Phänomenologie gibt, konnten aber Unterschiede in der affektiven Reaktion auf die Stimmen finden.

Auch einzelne Subskalen des BAVQ-R wurden in verschiedenen Untersuchungen verwendet. Für Böswilligkeit wurde im Rahmen einer Reliabilitätsprüfung der Subskala in einer norwegischen Studie $(N=31)$ ein Zusammenhang mit Aufmerksamkeitsdefiziten [12], und in einer Studie, die Korrelationen mit selbstverletzendem Verhalten bei Personen mit Schizophrenie und verbalen akustischen Halluzinationen untersuchte [13], eine positive Korrelation gezeigt. Validierte Übersetzungen liegen bisher in Französisch $(N=76)$ [14], Chinesisch $(N=33)$ [15] und Japanisch $(N=30)$ [16] vor.

Die vorliegende Studie prüft den Einsatz einer deutschen Version des BAVQ-R [1] an 93 stimmenhörenden PatientInnen mit Diagnosen von Psychosen aus dem schizophrenen Formenkreis.

\section{Methoden}

\section{Prozedere}

Der BAVQ-R [1] wurde durch unsere Arbeitsgruppe ins Deutsche übersetzt, die semantische Äquivalenz wurde durch Rückübersetzung durch eine zweisprachige englische Muttersprachlerin ermittelt, und dann durch einen der Autoren der englischen Originalversion, Paul Chadwick, geprüft und genehmigt.

Die Rekrutierung fand im Rahmen eines umfangreichen Projektes zu genderspezifischen Aspekten des Stimmenhörens, in welchem PatientInnen mit Erkrankungen aus dem schizophrenen Formenkreis (F2-Diagnose nach ICD10 [17]) rekrutiert wurden, statt. Weitere Einschlusskriterien neben der F2 Diagnose waren die Vollendung des 18. Lebensjahres sowie die Wahrnehmung von verbalen akustischen Halluzinationen innerhalb der letzten 4 Wochen vor Befragung. Als Ausschlusskriterien galten unzureichende Deutschkenntnisse und die Unfähigkeit die Einverständniserklärung zu erbringen.

Alle Prozeduren erfolgten nach den ethischen Standards entsprechend der Helsinki Deklaration. Nach Bewilligung durch die Ethikkommission der Medizinischen Universität Wien und die niederösterreichische Ethikkommission erfolgte die Rekrutierung an der Universitätsklinik für Psychiatrie und Psychothera- 
pie der Medizinischen Universität Wien und an der Universitätsklinik Tulln (stationäres und tagesklinisches Setting), am sozialpsychiatrischen Zentrum der Caritas Wien und den Wiener Psychosozialen Diensten (ambulantes Setting). Die PatientInnen erhielten ausführliche Informationen über die geplante Befragung, eine Teilnahme an der Studie erforderte die Unterzeichnung einer schriftlichen Einverständniserklärung. Den TeilnehmerInnen stand es frei den Ort für die Befragung (Institution oder zu Hause) zu wählen.

\section{Erhebungsinstrumente}

\section{Soziodemographische und klinische Variablen}

Für die Datenakquirierung von soziodemographischen Merkmalen und der Krankheitsanamnese (Ersterkrankungsalter, Anzahl früherer psychiatrischer stationärer Aufnahmen, Alter bei erster stationärer Behandlung) wurde ein eigens erstellter Fragebogen verwendet.

\section{The Beliefs About Voices Questionnaire - Revised} (BAVQ-R) [1]

Der BAVQ-R [1] ist ein Selbsteinschätzungsfragebogen, welcher aus 35 Items besteht. Durch fünf Subskalen (Böswilligkeit, Wohlwollen, Omnipotenz, Widerstand und Bindung) können Details über Überzeugungen, Emotionen und Verhaltensweisen bezogen auf die wahrgenommene Stimme erfasst werden. Antworten werden mittels 4-Punkt-Likert-Skala ( $0=$ stimme nicht $\mathrm{zu}, 1=$ weiß nicht, $2=$ stimme eher zu, 3=stimme zu) erfasst. Im Falle dessen, dass StudienteilnehmerInnen mehr als eine Stimme vernehmen, wird der Fragebogen für die dominante Stimme ausgefüllt.

\section{Psychotic Symptoms Rating Scale - Auditory} Hallucinations (PSYRATS-AH) [18, 19]

Die PSYRATS-AH [18] ist ein semi-strukturiertes Interview, in dem mittels 11 Items Details über das Stimmenhören (Häufigkeit, Dauer, Lokalisierung, Lautstärke, Überzeugung bzgl. Herkunft, Grad und Menge negativer Inhalte, Menge und Intensität von Leid durch die Stimmen, Lebensbeeinträchtigung, Kontrolle über die Stimme) erfasst werden. Der Fragebogen ist 5-Punkt-ordinalskaliert, nummeriert von 0-4.

\section{Clinical Global Impression Scale - Schizophrenia (CGI-SCH) [20]}

Die CGI-SCH [20] erfasst Negativ- und Positivsymptomatik, depressive und kognitive Symptome sowie einen Gesamtschweregrad einer Erkrankung. Jede der genannten Kategorien wird auf einer 7-Punkt-ordinalskalierten Skala $(1=$ nicht krank, $7=$ sehr schwer erkrankt) erfasst.

\section{Datenanalyse}

Alle statistischen Analysen wurden mittels „Statistical Package for Social Science“ (Version 22, SPSS Incl., IBM Corporation, USA) durchgeführt. Die Stichprobe wurde deskriptivstatistisch mittels der Angabe von Absolutwerten und Prozentwerten beschrieben.

Zur Analyse der Reliabilität wurde die Interne Konsistenz (Cronbach's alpha) herangezogen, wobei hier Werte über 0,7 als akzeptabel gelten. Die Test-RetestReliabilität wurde mittels Pearson-Korrelation berechnet, auch hier gelten Werte über 0,7 als akzeptabel.

Hinsichtlich Konstruktvalidität wurden PearsonKorrelationen zur Beurteilung der Ähnlichkeit bzw. Unterschiedlichkeit mit verschiedenen Konstrukten berechnet. Diese wurden für alle Subskalen des BAVQ-R [1] errechnet, wobei mögliche Zusammenhänge mit einzelnen Dimensionen des PSYRATS-AH [18] und der CGI-SCH [20] interessierten.

\section{Ergebnisse}

\section{Rekrutierung}

Zwischen Februar 2014 und Februar 2017 wurden insgesamt 117 in Frage kommende PatientInnen eingeladen an der Studie teilzunehmen. Davon gaben 93 Personen ihr Einverständnis und konnten somit in die Studie eingeschlossen werden. Die Diagnosen lauteten wie folgt: Paranoide Schizophrenie (F20.0) $(N=84)$, und Schizo-affektive Störung (F25.9) $(N=9)$. Um die Test-Retest-Reliabilität zu prüfen, füllten 19 StudienteilnehmerInnen nach 7-15 Tagen (Mittelwert: 9 Tage $\pm 2,24$ ) den Fragebogen ein zweites Mal aus.

\section{Stichprobe}

$56 \%$ der TeilnehmerInnen waren männlich, das mediane Alter des Samples betrug 33,0 Jahre (Spannweite: 19,0-84,0). 78,5\% der Teilnehmenden waren alleinstehend, nur 3\% waren berufstätig. Das Alter bei Ersterkrankung wurde von den TeilnehmerInnen mit 21 Jahren (Spannweite: 4-48) angegeben, die Dauer der Erfahrung des Stimmenhörens mit 10,5 Jahren (Spannweite: 4-48).

\section{Deskriptive Statistik des BAVQ-R}

Der Fragebogen konnte zügig, mit einem Zeitaufwand von unter $10 \mathrm{~min}$ ausgefüllt werden.

Tab. 1 zeigt die deskriptive Statistik der einzelnen Items und Subskalen des BAVQ-R. Hier wird ersichtlich, dass alle Subskalen Werte unterhalb des Mittelpunktes aufweisen. Daraus kann man schließen, dass die Fragen eher mit „ich stimme nicht zu“ oder ,ich weiß es nicht" oder (Werte 0 und 1 auf der LikertSkala) beantwortet wurden. 
Tab. 1 Deskriptive Statistik des BAVQ-R

\begin{tabular}{|c|c|c|c|c|}
\hline Items & $N$ & Mittelwert & Standardabweichung & Fehlend in \% \\
\hline Böswilligkeit & 89 & 7,9 & 5,39 & 4,3 \\
\hline Meine Stimme bestraft mich für etwas, das ich getan habe & 91 & 1,2 & 1,18 & 2,2 \\
\hline Meine Stimme verfolgt mich ohne guten Grund & 91 & 1,6 & 1,13 & 2,2 \\
\hline Meine Stimme ist böse & 91 & 1,6 & 1,26 & 2,2 \\
\hline Meine Stimme möchte mir Schaden zufügen & 91 & 1,4 & 1,27 & 2,2 \\
\hline Meine Stimme möchte, dass ich böse Dinge tue & 91 & 1,0 & 1,23 & 2,2 \\
\hline Meine Stimme versucht, mich zu verderben oder zu vernichten & 89 & 1,2 & 1,25 & 4,3 \\
\hline Wohlwollen & 89 & 5,0 & 5,47 & 4,3 \\
\hline Meine Stimme möchte mir helfen & 91 & 1,0 & 1,09 & 2,2 \\
\hline Meine Stimme möchte mich beschützen & 90 & 0,9 & 1,14 & 3,2 \\
\hline Meine Stimme hilft mir dabei, bei gutem Verstand zu bleiben & 91 & 0,8 & 1,13 & 2,2 \\
\hline $\begin{array}{l}\text { Meine Stimme unterstützt mich dabei, meine besonderen Kräfte oder } \\
\text { Fähigkeiten zu entwickeln }\end{array}$ & 91 & 0,9 & 1,06 & 2,2 \\
\hline Meine Stimme hilft mir, mein Lebensziel zu erreichen & 91 & 0,6 & 1,06 & 2,2 \\
\hline Ich bin dankbar, meine Stimme zu haben & 90 & 0,8 & 1,07 & 3,2 \\
\hline Omnipotenz & 87 & 8,9 & 3,95 & 6,5 \\
\hline Meine Stimme ist sehr mächtig & 90 & 1,8 & 1,19 & 3,2 \\
\hline Meine Stimme scheint alles über mich zu wissen & 91 & 2,2 & 1,02 & 2,2 \\
\hline Meine Stimme bringt mich dazu Dinge zu tun, die ich wirklich nicht tun will & 89 & 0,9 & 1,18 & 4,3 \\
\hline Ich kann meine Stimme nicht kontrollieren & 90 & 2,1 & 1,05 & 3,2 \\
\hline $\begin{array}{l}\text { Meine Stimme wird mir schaden oder mich töten, wenn ich ihr nicht } \\
\text { gehorche oder mich ihr widersetze }\end{array}$ & 90 & 0,7 & 1,09 & 3,2 \\
\hline Meine Stimme beherrscht mein Leben & 90 & 1,3 & 1,19 & 3,2 \\
\hline Widerstand & 88 & 13,1 & 7,53 & 5,4 \\
\hline Widerstand - Emotion & 88 & 5,9 & 3,96 & 5,4 \\
\hline Meine Stimme macht mir Angst & 90 & 1,5 & 1,20 & 3,2 \\
\hline Meine Stimme macht mich niedergeschlagen & 90 & 1,5 & 1,19 & 3,2 \\
\hline Meine Stimme macht mich wütend & 89 & 1,4 & 1,18 & 4,3 \\
\hline Meine Stimme macht mich ängstlich & 89 & 1,5 & 1,21 & 4,3 \\
\hline Widerstand - Verhalten & 90 & 7,2 & 4,75 & 3,2 \\
\hline \multicolumn{5}{|l|}{ Im Allgemeinen, wenn ich meine Stimme höre, } \\
\hline ... sage ich ihr, sie soll mich in Ruhe lassen & 90 & 1,3 & 1,33 & 3,2 \\
\hline ... versuche ich, mich abzulenken & 90 & 1,8 & 1,20 & 3,2 \\
\hline ... versuche ich, sie zu stoppen & 90 & 1,3 & 1,25 & 3,2 \\
\hline ... tue ich Dinge, um sie vom Sprechen abzuhalten & 90 & 1,2 & 1,26 & 3,2 \\
\hline ... sträube ich mich, ihr zu gehorchen & 90 & 1,5 & 1,24 & 3,2 \\
\hline Bindung & 89 & 6,2 & 6,25 & 4,3 \\
\hline Bindung - Emotion & 89 & 2,7 & 3,51 & 4,3 \\
\hline Meine Stimme gibt mir Sicherheit & 90 & 0,8 & 1,04 & 3,2 \\
\hline Meine Stimme macht mich glücklich & 90 & 0,6 & 1,03 & 3,2 \\
\hline Meine Stimme macht mich ruhig & 89 & 0,7 & 1,03 & 4,3 \\
\hline Meine Stimme macht mich zuversichtlich & 90 & 0,7 & 1,02 & 3,2 \\
\hline Bindung - Verhalten & 89 & 3,4 & 3,31 & 4,3 \\
\hline \multicolumn{5}{|l|}{ Im Allgemeinen, wenn ich meine Stimme höre, } \\
\hline ... höre ich ihr zu, weil ich es möchte & 89 & 1,2 & 1,21 & 4,3 \\
\hline ... befolge ich bereitwillig, was meine Stimme mir befiehlt & 89 & 0,6 & 0,96 & 4,3 \\
\hline ... habe ich Dinge getan um mit meiner Stimme in Kontakt zu treten & 90 & 1,0 & 1,15 & 3,2 \\
\hline ... suche ich Rat bei meiner Stimme & 90 & 0,8 & 1,14 & 3,2 \\
\hline
\end{tabular}


Tab. 2 Interne Konsistenz der BAVQ-R Subskalen

\begin{tabular}{|l|l|}
\hline Subskala & Cronbach's Alpha \\
\hline Böswilligkeit & 0,832 \\
\hline Wohlwollen & 0,910 \\
\hline Omnipotenz & 0,619 \\
\hline Widerstand & 0,852 \\
\hline Widerstand-Emotion & 0,841 \\
\hline Widerstand - Verhalten & 0,815 \\
\hline Bindung & 0,872 \\
\hline Bindung-Emotion & 0,875 \\
\hline Bindung-Verhalten & 0,725 \\
\hline
\end{tabular}

Tab. 3 Test-Retest-Reliabilität

\begin{tabular}{|l|l|l|}
\hline Subskala & $\begin{array}{l}\text { Korrelation nach } \\
\text { Pearson }\end{array}$ & Signifikanz \\
\hline Böswilligkeit & 0,883 & $p \leq 0,01$ \\
\hline Wohlwollen & 0,775 & $p \leq 0,01$ \\
\hline Omnipotenz & 0,858 & $p \leq 0,01$ \\
\hline Widerstand & 0,701 & $p=0,001$ \\
\hline Widerstand - Emotion & 0,610 & $p=0,007$ \\
\hline Widerstand - Verhalten & 0,760 & $p \leq 0,01$ \\
\hline Bindung & 0,466 & $p=0,051$ \\
\hline Bindung-Emotion & 0,672 & $p=0,002$ \\
\hline Bindung-Verhalten & 0,542 & $p=0,017$ \\
\hline
\end{tabular}

\section{Interne Konsistenz}

Zufriedenstellende interne Konsistenzen konnten für die Subskalen Böswilligkeit $(\alpha=0,83)$, Wohlwollen $(\alpha=0,91)$, Widerstand $(\alpha=0,85)$, Widerstand Emotion $(\alpha=0,84)$, Widerstand - Verhalten $(\alpha=0,82)$, Bindung $(\alpha=0,87)$, Bindung - Emotion $(\alpha=0,88)$ und Bindung - Verhalten $(\alpha=0,73)$ gezeigt werden. Lediglich die Subskala Omnipotenz zeigte mit $\alpha=0,62$ einen Wert unterhalb der Akzeptanzgrenze von 0,7. Die Ergebnisse werden in Tab. 2 dargestellt.

\section{Test-Retest-Reliabilität}

In Tab. 3 wird ersichtlich, dass die Subskalen Böswilligkeit, Wohlwollen, Omnipotenz, und Widerstand sowie die Subkategorie Widerstand-Verhalten die Akzeptanzgrenze von 0,7 erreichen. Die Subskala Bindung sowie deren Subkategorien Bindung-Emotion, Bindung-Verhalten und die Subkategorie WiderstandEmotion erreichen den Wert von 0,7 nicht.

\section{Konstruktvalidität}

Zur Ermittlung der Konstruktvalidität wurden Pearson-Korrelationen zur Beurteilung der Ähnlichkeit bzw. Unterschiedlichkeit der Konstrukte des BAVQ-R mit verschiedenen Dimensionen von PSYRATS-AH sowie CGI-SCH und dem Depressionsitem der CGI-SCH berechnet. Analysen wurden für alle Subskalen des BAVQ-R durchgeführt. Die Subskala „Böswilligkeit“ zeigte signifikante Korrelationen mit den PSYRATSAH Items „negative Inhalte“ $(\mathrm{r}=0,40 ; p \leq 0,01)$, „Höhe der Belastung“ ( $\mathrm{r}=0,41 ; p \leq 0,01)$, der „Intensität der Belastung“ ( $\mathrm{r}=0,33 ; p=0,002)$ sowie mit der „Dauer“ $(\mathrm{r}=0,21 ; p=0,046)$.

Bei der Subskala „Wohlwollen“ gab es signifikante negative Korrelationen mit den Items des PSYRATSAH „negative Inhalte“ $(\mathrm{r}=-0,43 ; p \leq 0,01)$ und „Höhe der Belastung“ ( $\mathrm{r}=-0,40 ; p \leq 0,01)$ sowie mit dem Item „Intensität der Belastung“ ( $\mathrm{r}=-0,39 ; p \leq 0,01)$ und „Dauer“ ( $\mathrm{r}=-0,21 ; p=0,05)$ und Lautstärke $(\mathrm{r}=-0,25$; $p=0,02)$.

Die Subskala „Omnipotenz“ des BAVQ-R zeigte signifikante Korrelationen mit „Dauer“ ( $\mathrm{r}=0,38 ; p \leq 0,01)$, „negativen Inhalten“ $(\mathrm{r}=0,36 ; p=0,001)$, „Höhe der Belastung“ ( $\mathrm{r}=0,34 ; p=0,001)$ und „Intensität der Belastung“ ( $\mathrm{r}=0,34 ; p=0,002)$, „Häufigkeit“ ( $\mathrm{r}=0,25$; $p=0,018)$ und „Kontrolle“ ( $\mathrm{r}=0,24 ; p=0,028)$.

Die Subskala Widerstand korrelierte mit „Höhe der Belastung“ ( $\mathrm{r}=0,54 ; p \leq 0,01)$, „Intensität der Belastung“ ( $\mathrm{r}=0,46 ; p \leq 0,01)$, „negative Inhalte“ $(\mathrm{r}=0,34$; $p=0,001)$, „Dauer“ $(\mathrm{r}=0,27 ; p=0,011)$ und „Lautstärke“ ( $\mathrm{r}=0,22 ; p=0,041)$.

Die Subskala „Bindung“ korrelierte negativ mit „negative Inhalte“ $(\mathrm{r}=-0,40 ; p \leq 0,01)$, „Höhe der Belastung“ ( $\mathrm{r}=-0,33 ; p=0,002)$, „Intensität der Belastung“ $(\mathrm{r}=-0,35 ; p=0,001)$, „Kontrolle“ $(\mathrm{r}=-0,36 ; p=0,001)$ und „Lautstärke“ ( $\mathrm{r}=-0,23 ; p=0,028)$.

\section{Korrelationen der Subskalen untereinander}

Die Subskala „Böswilligkeit“ zeigte Korrelationen mit den Subskalen „Widerstand“ ( $\mathrm{r}=0,596 ; p<0,01)$, „Omnipotenz" $(\mathrm{r}=0,493 ; p<0,01)$, sowie negative Korrelationen mit „Wohlwollen“ $(\mathrm{r}=-0,564 ; p<0,01)$ und „Bindung“ ( $\mathrm{r}=-0,407 ; p<0,01)$.

Bei der Subskala „Wohlwollen“ bestanden Korrelationen mit der Subskala „Bindung“ ( $\mathrm{r}=0,856$; $p<0,01)$, sowie negative Korrelationen mit „Böswilligkeit“ ( $\mathrm{r}=-0,564 ; p<0,01)$, „Widerstand“ $(\mathrm{r}=-0,588$; $p<0,01)$ und „Omnipotenz" ( $\mathrm{r}=-0,261 ; p=0,015)$.

Die Subskala „Omnipotenz“ zeigte Korrelationen mit den Subskalen „Widerstand“ $(\mathrm{r}=0,370 ; p<0,01)$, „Böswilligkeit“ ( $\mathrm{r}=0,493 ; p<0,01)$, sowie negative Korrelationen mit „Wohlwollen“ $(\mathrm{r}=-0,261 ; p=0,015)$ und „Bindung“ ( $\mathrm{r}=-0,211 ; p=0,050)$.

Die Subskala Widerstand korrelierte mit der Subskala Bindung $(\mathrm{r}=0,484 ; p<0,01)$.

\section{Korrelationen mit der CGI-SCH [20]}

Der Mittelwert der CGI-SCH lag im vorliegenden Sample bei 3,97 $\pm 0,86$. Es ergaben sich keine Korrelationen zwischen den Subskalen und dem Schweregrad der Erkrankung. Weder mit dem Gesamtscore der CGI$\mathrm{SCH}$, noch mit dem Item Depressivität. 


\section{Diskussion}

Die vorliegende Stichprobe ist hinsichtlich der typischen sozialen und beruflichen Beeinträchtigungen repräsentativ für über längere Zeit an Schizophrenie erkrankte Personen [21]. Die Ergebnisse zeigten eine hohe Quote an alleinstehenden Personen sowie Arbeitslosigkeit und ein schlechtes soziales Netzwerk.

Der Fragebogen erweist sich hier als praktikabel für die untersuchte Personengruppe, da er selbstständig, großteils vollständig und zügig ausgefüllt werden konnte. Wie auch in der französischen Validierungsstudie von Zanello et al. [13], zeigt er sich sowohl inhaltlich als auch hinsichtlich des Zeitaufwandes als geeignet.

Die vorliegende Stichprobe ist mit einer Größe von $N=93$ die bisher größte Fallzahl in einer BAVQ-R Validierungsstudie. Die Studie wurde ausschließlich mit Personen mit Erkrankungen aus dem schizophrenen Formenkreis durchgeführt. In die chinesische Validierungsstudie $(N=33)$ [15] und die japanische Validierungsstudie $(N=30)$ [16] wurden ebenfalls ausschließlich Personen mit F2-Erkrankungen einbezogen. In der Originalstudie $(N=71)$ [1] und der französischen Validierungsstudie $(N=76)$ [14] bestand die jeweilige Stichprobe aus Personen mit F2 und F3 Diagnosen nach ICD-10 [17].

Wie in der Originalstudie [1] zeigte in der vorliegenden Studie die Subskala Widerstand eher hohe Werte und die Subskala Wohlwollen eher niedrige Werte. Dies legt die Vermutung nahe, dass viele der TeilnehmerInnen ihre Stimme als nicht wohlwollend und als emotional belastend erlebten. In der Subskala Omnipotenz können einerseits sehr variable Mittelwerte zwischen den einzelnen Items, andererseits auch die höchste Anzahl an fehlenden Antworten (6,5\%) über die gesamte Subskala gesehen werden. Über die Items der anderen Subskalen verlaufen die Mittelwerte weitgehend stabil.

Ähnlichkeiten mit der Originalvalidierungsstudie [1] zeigten sich auch hinsichtlich der internen Konsistenz. In der Originalstudie wurden zwar für alle Subskalen akzeptable Werte erzielt, waren jedoch in der Subskala Omnipotenz mit $r=0,74$ eher niedrig. In der vorliegenden Studie konnte die Subskala Omnipotenz im Gegensatz zu den anderen Subskalen den akzeptablen Wert von 0,7 nicht erreichen. In der französischen Studie von Zanello et al. [14] zeigte die Subskala Omnipotenz ebenfalls einen Wert unter der Akzeptanzgrenze $(r=0,64)$, ähnlich der vorliegenden Studie $(r=0,62)$. Diese Ergebnisse geben Anlass die Aussagen der Omnipotenzskala nochmals zu überdenken. Kürzlich wurde in England von Strauss et al. [22] eine Faktorenanalyse mit gepoolten Daten aus unterschiedlichen Studien $(N=450)$ durchgeführt, welche zu dem Ergebnis kam, dass die Skala modifiziert werden könnte, da die Subskalen Omnipotenz und Böswilligkeit zu einem Konstrukt ,Verfolgungsüberzeugungen' zusammengefasst werden könnten.
In dem speziellen Datenset von Strauss et al. [22] wurde auch die Unterscheidung zwischen emotionalen und Verhaltensreaktionen sowohl in Bezug auf Bindung als auch in Bezug auf Widerstand in Frage gestellt. Die Subskala Bindung konnte in dem vorliegenden Subsample zur Test-Retest-Reliabilität als einzige Subskala keine signifikante Korrelation erreichen, die Subskala Widerstand konnte den akzeptablen Wert von 0,7 nur sehr knapp erreichen. Dies legt die Vermutung nahe, dass Überzeugungen bezüglich der Stimme zeitlich stabil sind, aber Emotionen und Verhalten im Bezug auf Bindung und Widerstand nicht so stabil sind. Die Subkategorien Bindung-Emotion und Bindung-Verhalten zeigten bei separater Betrachtung jeweils unterschiedliche, unter $r=0,7$ (Pearson) liegende, aber signifikant korrelierte Werte. Dieses Ergebnis könnte in zukünftigen Erhebungen geprüft werden und im Rahmen der Entwicklungen der dritten Welle der kognitiven Verhaltenstherapie, die die Metakognitionen und deren Einfluss auf das Verhalten in den Vordergrund stellt [23], diskutiert werden, und mit den Schlussfolgerungen einer PSYRATS-Studie von Kronmüller et al. [24] in Beziehung gesetzt werden, in welcher gefunden wurde, dass akustische Halluzinationen über das 4-Faktoren-Modell (Kognitive Interpretation, emotionale und Kontrollcharakteristika sowie körperliche Charakteristika).

Es konnten keinerlei Korrelationen mit dem generellen Schweregrad (CGI-SCH-Gesamt und CGIDepressivität [20]) von Erkrankung und Depressivität gefunden werden. Mit mehr differenzierten Maßen zu einzelnen Aspekten der Symptomatik und ihren Schweregraden würden möglicherweise Zusammenhänge zu zeigen sein, wie es in der Originalstudie für depressive und Angstsymptome gemessen mit der Hospital Anxiety and Depression Scale [25] der Fall war.

In der Originalstudie, wie auch in der vorliegenden Untersuchung jedoch, wurden die theoretisch nachvollziehbaren positiven Korrelationen von Belastung und negativen Inhalten mit Böswilligkeit und Widerstand einerseits und negativen Korrelationen mit Wohlwollen und Bindung andererseits gefunden.

Auch in der Zusammenschau mit den Korrelationen zwischen den Subskalen wurde deutlich, dass Überzeugungen zur Böswilligkeit und Omnipotenz der Stimmen die Widerstandskräfte der Stimmenhörer strapazieren, während Überzeugungen zum Wohlwollen der Stimmen und Bindung positiv korreliert dem gegenüberstehen.

Mehr als vom Schweregrad der schizophrenen Erkrankung oder dem Ausmaß der Depressivität scheint hier also die Belastung und das resultierende Verhalten von den Überzeugungen über Macht und Intention der gehörten Stimmen abhängig. Insgesamt weisen die Ergebnisse darauf hin, dass der Fragebogen BAVQ- $R$ ein robustes zugrundeliegendes Konzept ab- 
bildet, das auch von den ausfüllenden Personen so wahrgenommen wird.

\section{Limitationen}

In der vorliegenden Studie wurden Personen mit einer durchschnittlichen Stimmenerfahrung von 10,5 Jahren (Spannweite: 0,1-45 Jahre) eingebunden. Aus diesem Grund erlauben es unsere Ergebnisse nicht, Aussagen über Patientengruppen wie Ersterkrankte zu treffen. Weiters suggerieren die erhobenen Daten der Clinical Global Impression Scale - Gesamtscore [20] $(N=89$, Mittelwert: 3,97 $\pm 0,86)$, dass das Sample einen mittelschweren Erkrankungsgrad aufwies und somit keine Aussagen über die Praktikabilität des BAVQ-R bei schwerstkranken Personen zulässt. Da in diese Studie nur Personen mit F2-Erkrankungen einbezogen wurden, können keine Aussagen über stimmenhörende Personen mit anderen Diagnosen wie z.B. affektive Störungen, post-traumatischen Belastungsstörungen oder gesunde StimmenhörerInnen getroffen werden. Auch liegen leider keine Daten zum Substanzkonsum vor, was gerade in der untersuchten Population und Symptomatik relevant erscheint.

\section{Schlussfolgerungen für die Praxis}

Die einzuschätzenden Dimensionen bilden relevante Aspekte des subjektiven Erlebens des Stimmenhörens ab. Sowohl aus therapeutischer Sicht als auch als Forschungsbedarf stellt sich weiterhin aktuell die Frage nach der Konzeptualisierung vom Zusammenhang zwischen Kognition, Emotion und Verhalten.

Danksagung Die Autoren danken allen Personen, die an der Studie teilgenommen haben. Besonderen Dank möchten wir auch Ingrid Sibitz für ihren wertvollen Beitrag und Einsatz in den Anfängen des Projektes aussprechen. Weiters danken wir dem Sozialpsychiatrischen Zentrum der Caritas Wien, den Psychosozialen Diensten Wien (PSD) und der Abteilung für Erwachsenenpsychiatrie der Universitätsklinik Tulln für ihre Hilfe bei Rekrutierung.

Förderung Diese Arbeit wurde durch den Jubiläumsfond der Österreichischen Nationalbank gefördert (Ref: 16390).

Funding Open access funding provided by Medical University of Vienna.

Interessenkonflikt A. Gmeiner, J. Aslan, A. Gaglia, T. Rumpold, B. Schrank, S. Süßenbacher und M. Amering geben an, dass kein Interessenkonflikt besteht.

Open Access Dieser Artikel wird unter der Creative Commons Namensnennung 4.0 International Lizenz (http:// creativecommons.org/licenses/by/4.0/deed.de) veröffentlicht, welche die Nutzung, Vervielfältigung, Bearbeitung, Verbreitung und Wiedergabe in jeglichem Medium und Format erlaubt, sofern Sie den/die ursprünglichen Autor(en) und die Quelle ordnungsgemäß nennen, einen Link zur Creative Commons Lizenz beifügen und angeben, ob Änderungen vorgenommen wurden.

\section{Literatur}

1. Chadwick P, Lees S, Birchwood M. The revised Beliefs About Voices Questionnaire (BAVQ-R). Br J Psychiatry. 2000;177:229-32.

2. Chadwick P, Birchwood M. The omnipotence of voices. II: the Beliefs About Voices Questionnaire (BAVQ). Br J Psychiatry. 1995;166(6):773-6.

3. Chadwick P, Birchwood M. The omnipotence of voices. A cognitive approach to auditory hallucinations. Br J Psychiatry. 1994;164(2):190-201.

4. Craig T, Ward T, Rus-Calafell M. Wellcome trust-funded monographs and book chapters. AVATAR therapy for refractory auditory hallucinations. In: Pradhan B, Pinninti N, Rathod S, Hrsg. Brief interventions for psychosis: a clinical compendium. London: Springer; 2016.

5. Leff J, Williams G, Huckvale MA, Arbuthnot M, Leff AP. Computer-assisted therapy for medication-resistant auditory hallucinations: proof-of-concept study. Br J Psychiatry. 2013;202:428-33. https://doi.org/10.1192/bjp.bp.112. 124883.

6. Birchwood M, Michail M, Meaden A, Tarrier N, Lewis $\mathrm{S}$, Wykes T, et al. Cognitive behaviour therapy to prevent harmful compliance with command hallucinations (COMMAND): a randomised controlled trial. LancetPsychiatry. 2014;1(1):23-33. https://doi.org/10.1016/s22150366(14)70247-0.

7. Hartigan N, McCarthy-Jones S, Hayward M. Hear today, not gone tomorrow? An exploratory longitudinal study of auditory verbal hallucinations (hearing voices). Behav Cogn Psychother. 2014;42(1):117-23. https://doi.org/10. 1017/s1352465813000611.

8. Penn DL, Meyer PS, Evans E, Wirth RJ, Cai K, Burchinal M. A randomized controlled trial of group cognitive-behavioral therapyvs. enhanced supportive therapy for auditoryhallucinations. Schizophr Res. 2009;109(1-3):52-9. https://doi. org/10.1016/j.schres.2008.12.009.

9. EllettL, Luzon O, Birchwood M, AbbasZ, Harris A, Chadwick P. Distress, omnipotence, and responsibility beliefs in command hallucinations. Br J Clin Psychol. 2017;56(3):303-9. https://doi.org/10.1111/bjc.12139.

10. Slotema CW, Blom JD, Deen M, Niemantsverdriet MBA, van der Gaag M, Hoek HW, et al. Negative beliefs about voices in patients with borderline personality disorder are associated with distress: a plea for cognitive-behavioural therapy? Psychopathology. 2017;50(4):255-61. https://doi. org/10.1159/000477669.

11. Hepworth CR, Ashcroft K, Kingdon D. Auditory hallucinations: a comparison of beliefs about voices in individuals with schizophrenia and borderline personality disorder. Clin Psychol Psychother. 2013;20(3):239-45. https://doi.org/10. 1002/cpp.791.

12. KrakvikB, Stiles T, Hugdahl K. Experiencing malevolent voices is associated with attentional dysfunction in psychotic patients. Scand J Psychol. 2013;54(2):72-7. https://doi.org/ 10.1111 /sjop. 12024 .

13. Simms J, McCormack V, Anderson R, Mulholland C. Correlates of self-harm behaviour in acutely ill patients with schizophrenia. Psychol Psychother. 2007;80(Pt 1):39-49. https://doi.org/10.1348/147608306x99386.

14. Zanello A, Badan Ba M. Validation of the French version of the "Beliefs About Voices Questionnaire-Revised" (BAVQR). Encephale. 2016;42(4):320-4. https://doi.org/10.1016/ j.encep.2016.03.006.

15. Wong AW, Chen EY. Psychometric properties of translated outcome measures of cognitive behavioural therapy 
for psychosis in the Chinese context. Asian J Psychiatr. 2015;13:62-5.https://doi.org/10.1016/j.ajp.2014.09.005.

16. Kaneda Y. Psychometric properties of the Japanese version of the beliefs about voices questionnaire-revised. 2008.

17. Organization WH. The ICD-10 classification of mental and behavioural disorders: clinical descriptions and diagnostic guidelines. Geneva. World Health Organization; 1992.

18. Haddock G, McCarron J, Tarrier N, Faragher EB. Scales to measure dimensions of hallucinations and delusions: the psychotic symptom rating scales (PSYRATS). Psychol Med. 1999;29(4):879-89.

19. Schneider SD, Jelinek L, Lincoln TM, Moritz S. What happened to the voices? A fine-grained analysis of how hallucinations and delusions change under psychiatric treatment. Psychiatry Res. 2011;188(1):13-7.https://doi.org/10.1016/ j.psychres.2010.12.013.

20. Haro J, Kamath S, Ochoa S, Novick D, Rele K, Fargas A, et al. The Clinical Global Impression-Schizophrenia scale: a simple instrument to measure the diversity of symptoms present in schizophrenia. Acta Psychiatr Scand. 2003;107:16-23.
21. Couture SM, Penn DL, Roberts DL. The functional significance of social cognition in schizophrenia: a review. Schizophr Bull. 2006;32(Suppl 1):S44-S63. https://doi.org/ $10.1093 / \mathrm{schbul} / \mathrm{sbl} 029$.

22. Strauss C, Hugdahl K, Waters F, Hayward M, Bless JJ, Falkenberg LE, et al. The beliefs about voices questionnaire-revised:afactorstructurefrom 450 participants. Psychiatry Res. 2018;259:95-103. https://doi.org/10.1016/ j.psychres.2017.09.089.

23. Hayes SC, Hofmann SG. The third wave of cognitive behavioral therapy and the rise of process-based care. World Psychiatry.2017;16(3):245-6.https://doi.org/10.1002/wps. 20442.

24. Kronmuller KT, von BockA, Grupe S, Buche L, Gentner NC, RucklS, etal.Psychometric evaluation of the psychoticsymptom rating scales. Compr Psychiatry. 2011;52(1):102-8. https://doi.org/10.1016/j.comppsych.2010.04.014.

25. ZigmondAS, Snaith RP.Thehospital anxiety and depression scale. Acta Psychiatr Scand. 1983;67(6):361-70. 